\title{
The Impact of Sleep on Female Sexual Response and Behavior: A Pilot Study
}

\author{
David A. Kalmbach, PhD, ${ }^{*}$ J. Todd Arnedt, PhD, ${ }^{\star}$ Vivek Pillai, $\mathrm{PhD},{ }^{\dagger}$ and Jeffrey A. Ciesla, $\mathrm{PhD}^{\ddagger}$ \\ *Sleep and Circadian Research Laboratory, Department of Psychiatry, University of Michigan Medical School, Ann

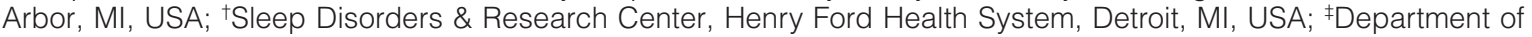 \\ Psychological Sciences, Kent State University, Kent, OH, USA
}

DOI: $10.1111 /$ jsm. 12858

\section{A B S T R A C T}

Introduction. The etiological role of sleep disturbance in sexual difficulties has been largely overlooked. Research suggests that short sleep duration and poor sleep quality lead to poor female sexual response. However, prior research consists of cross-sectional studies, and the influence of sleep on sexual functioning and behavior has not been prospectively examined.

Aim. We sought to examine the influence of nightly sleep duration, sleep quality, and sleep onset latency on daily female sexual response and activity.

Methods. This study used a longitudinal design to study 171 women free of antidepressants and with reliable Internet access who were recruited from a university setting in the United States. Participants first completed baseline measures in a laboratory, and then completed web-delivered surveys at their habitual wake time for 14 consecutive days.

Main Outcome Measures. All outcome measures were modified for daily recall. Participants completed the Profile of Female Sexual Function's desire, subjective arousal, and orgasmic functioning scales and the Female Sexual Function Index's genital arousal scale, and indicated whether they engaged in partnered sexual activity or selfstimulation in response to dichotomous items.

Results. Analyses revealed that longer sleep duration was related to greater next-day sexual desire $(b=0.32$, $P=0.02$ ), and that a 1 -hour increase in sleep length corresponded to a $14 \%$ increase in odds of engaging in partnered sexual activity (odds ratio $=1.14, P<0.05$ ). In contrast, sleeping longer predicted poorer next-day genital arousal $(b=-0.19, P<0.01)$. However, results showed that women with longer average sleep duration reported better genital arousal than women with shorter average sleep length $(b=0.54, P=0.03)$.

Conclusions. Obtaining sufficient sleep is important to the promotion of healthy sexual desire and genital response, as well as the likelihood of engaging in partnered sexual activity. These relationships were independent of daytime affect and fatigue. Future directions may investigate sleep disorders as risk factors for sexual dysfunction. Kalmbach DA, Arnedt JT, Pillai V, and Ciesla JA. The impact of sleep on female sexual response and behavior: A pilot study. J Sex Med 2015;12:1221-1232.

Key Words. Sleep Duration; Sleep Quality; Female Sexual Response; Sexual Dysfunction; Insomnia; Sleep Disturbance

\section{Introduction}

$\mathrm{T}$ he biopsychosocial model of female sexual response highlights the complexity of the factors underlying sexual dysfunction. Prior research has identified medical illness [1], psychological disorder [1-3], and relationship dissatisfaction [4] as risk factors for sexual dysfunctions, such as hypoactive desire or diminished sexual arousal. Though initially studied as early as the 1960s, the 
intersection of sleep and sexual functioning has since been largely overlooked. The literature on sleep and female sexuality is limited to a modest number of studies on rapid eye movement (REM) sleep and nocturnal vaginal vasocongestion from the 1960s through mid-1980s [5-7], then more recently on the roles of hormones in sexual function and sleep, and sleep-disordered breathing (e.g., obstructive sleep apnea) as a risk factor for sexual problems [8-10]. With growing recognition of the morbidity associated with disordered or insufficient sleep [11], it is surprising that the fields of sexology and sleep medicine have paid little attention to this area. As a step toward addressing this gap, we examined the influence of nightly sleep on sexual response and activity in young women.

In the 1960s, the occurrence of penile tumescence during REM sleep periods became the focus of much research, eventually aiding differential diagnosis between psychogenic and organic causes of erectile dysfunction [12]. These findings naturally led to an interest in sleep stage-related genital arousal changes in women. Researchers identified sleep-related changes in nocturnal vaginal vasocongestion such that, similar to nocturnal penile tumescence in men, increases in vaginal blood flow occurred during $95 \%$ of REM periods $[6,12]$. However, unlike men, women experienced a moderately high frequency of vaginal blood flow increases during non-REM periods ( $66 \%$ [6]). To this day, it remains unclear what function REM sleep plays, if any, in human sexual response. One theory from the 1970s, based on findings that chronic REM sleep deprivation in laboratory animals led to hypersexuality, proposed that REM sleep decreases waking drive-motivated behavior, such as appetitive sexual behavior [7,13]. This theory, however, has not been borne out in the literature.

More recently, Andersen and colleagues' qualitative review [12] offered that neuroendocrine reproductive control activity may mediate the influence of sleep on human sexual response. To support their theory, the authors attempted to marry two literatures: (i) sex hormones (namely androgens) and sexual behavior; and (ii) sex hormones and sleep. They noted that sufficient levels of testosterone, progesterone, and estrogen have all been identified as important to healthy sexual function (indicated by greater desire and arousability) and increased sexual behavior in women. Additionally, the authors highlighted that sleeping increases androgen levels, whereas waking is related to their reduction. Therefore, they hypothesized that one pathway from sleep loss or deprivation to impaired sexual function may be mediated by deficient androgen levels. They concluded their review by emphasizing the importance of sufficient sleep duration and sleep quality (SQ) as crucial to the maintenance of healthy sexual functioning in men and women.

Despite this growing body of research, a number of gaps exist in our understanding of the impact of sleep on female sexual response and behavior. Though it has been hypothesized that poor sleep duration and quality lead to greater difficulties with sexual function [12], no studies to date have prospectively examined this hypothesis. Experience sampling techniques would capture the manner in which nightly sleep experience is related to next-day sexual functioning and behavior. Additionally, though studies have demonstrated poorer sexual response in patients with untreated sleep-related breathing disorders (e.g., Köseoğlu et al. and other authors [8-10]), it is presently unclear if this association is directly sleep-related or due to confounding comorbid conditions. As such, it is important to investigate sleep and sexual function and behavior in a relatively healthy sample that is less likely to be confounded by medical, psychiatric, or sleep-related illnesses or disorders.

\section{Aims}

Using a 2-week daily diary approach, we characterized the manner in which nightly sleep predicted next-day sexual functioning and behavior in a sample of young adult women. To reduce potential confounds, we accounted for the influence of age, oral contraceptive use, baseline severity of sexual distress, anxiety, and depression. Additionally, as changes in sexual activity [14] and desire [15] have been associated with the menstrual cycle, the presence of menstruation was also examined as a possible confound. We also accounted for daily affect and fatigue to minimize the potential confounding influences of affect and fatigue on sleep and sexual response. Regarding affect, we specifically focused on affect balance, which is conceptualized as the ratio of positive to negative affect an individual experiences. That is, more important than how bappy or sad someone may be is how much happier than they were sad within a certain time period. Affect balance has been viewed as an overall measure of psychological well-being that has shown to be related to sexual functioning [16]. By using repeated assessments, we aimed to capture nightly variations in total sleep time (TST), SQ, 
and sleep onset latency (SOL) and their correspondence to day-to-day fluctuations in desire, arousal, genital response, and orgasmic function. We hypothesized that nights characterized by sleep loss (i.e., shorter TST), poorer SQ, and greater difficulty initiating sleep (i.e., longer SOL) would lead to poorer next-day sexual functioning.

\section{Methods}

\section{Participants}

Participants were recruited for a parent study investigating the relationship between female sexual response and mood. One hundred and seventy-one healthy female college students were assessed for a period of 14 days (see Table 1 for sample characteristics). Approximately half of the sample reported having a significant other; the average length of the relationship was over two and a half years, though substantial variability was observed. Further, more than half of the sample reported having at least one sexual partner at baseline. A little more than half of the women in this study reported taking some form of oral contraceptive. Participants were recruited from psychology courses at a Midwestern university and received course credit for their participation. Inclusion criteria were reliable Internet access and being free of antidepressants (to reduce medication-related iatrogenic effects) for at least 4 weeks prior to participation. The local institutional review board approved this study. All women provided written informed consent prior to participation.

\section{Procedure}

The study protocol involved a baseline assessment and 14 daily follow-up assessments. At baseline, participants reported demographic information and completed questionnaires about sexual distress, depression, and anxiety, and then received instructions on how to complete the daily, web-delivered questionnaires. Participants were instructed to complete all daily assessments on their home computer, in private, upon waking each morning at their habitual wake time. Questionnaires assessed affect and sexual function over the previous 24 hours, whereas sleep questions asked about participants' sleep experiences "last night."

\section{Baseline Measures}

Demographic information, relationship history, and oral contraceptive use were reported at baseline (see Table 1 for sample characteristics).

The Female Sexual Distress Scale-Revised (FSDS-R) [17] is a 13-item self-report questionnaire used to assess sex-related personal distress in women. In the present study, respondents indicated the extent to which they experienced sexual distress over the past month with higher scores indicating higher levels of sexual distress. Internal consistency in our sample was adequate $(\alpha=0.74)$.

The Center for Epidemiologic Studies Depression Scale (CESD) [18] is a 20-item self-report

Table 1 Sample descriptive characteristics $(N=171)$

\begin{tabular}{|c|c|}
\hline Age & $M \pm S D: 20.07 \pm 3.32$ \\
\hline \multicolumn{2}{|l|}{ Race } \\
\hline White & $n=139 ; 81.29 \%$ \\
\hline Black & $\mathrm{n}=23 ; 13.45 \%$ \\
\hline Eastern Asian or Pacific Islander & $\mathrm{n}=4 ; 2.34 \%$ \\
\hline Hispanic or Latino & $\mathrm{n}=2 ; 1.17 \%$ \\
\hline Western Asian or Middle Eastern & $\mathrm{n}=1 ; 0.58 \%$ \\
\hline "Other" & $\mathrm{n}=2 ; 1.17 \%$ \\
\hline Do you currently have a significant other? & "Yes" $n=86 ; 50.59 \%$ of 170 responders \\
\hline Length of relationship & $\mathrm{M} \pm \mathrm{SD}: 20.71$ months \pm 18.57 \\
\hline \multicolumn{2}{|l|}{ Sexual partner status } \\
\hline 0 partner & $\mathrm{n}=76 ; 44.44 \%$ \\
\hline 1 partner & $\mathrm{n}=92 ; 53.80 \%$ \\
\hline 2 partners & $\mathrm{n}=2 ; 1.17 \%$ \\
\hline 3 partners & $\mathrm{n}=1 ; 0.58 \%$ \\
\hline \multicolumn{2}{|l|}{ Sexual orientation } \\
\hline Bisexual, equally attracted to men and women & $\mathrm{n}=3 ; 1.75 \%$ \\
\hline Mostly or completely homosexual & $n=8 ; 4.68 \%$ \\
\hline Number of lifetime sexual partners & $\mathrm{M} \pm \mathrm{SD}$, range: $4.29 \pm 4.74,0-24$ \\
\hline Using oral contraceptives & $\mathrm{n}=97 ; 56.73 \%$ \\
\hline
\end{tabular}

$\mathrm{M}=$ mean; $\mathrm{SD}=$ standard deviation 
inventory used to measure symptoms of depression. It was modified to assess these symptoms over the past month. Scores on the CESD range from 0 to 60 with higher scores indicating greater depression severity. In the present sample, the CESD achieved high internal consistency $(\alpha=0.90)$.

State-Trait Anxiety Inventory Form X-State (STAIXS) [19] is a 20-item self-report inventory intended to assess levels of anxiety. It was modified to assess these symptoms over the past month. Possible scores on the STAIXS range from 20 to 80 with higher scores indicating greater anxiety severity. Internal consistency of the STAIXS was high $(\alpha=0.94)$ in the present sample.

\section{Main Outcome Measures Daily Measures}

The Profile of Female Sexual Function (PFSF) $[20,21]$ is a self-report tool assessing sexual response. It was modified for daily use in the present study and has been validated for use in nonclinical samples [22]. For the present study, women reported on their experiences of sexual desire $(\alpha=0.94)$, subjective sexual arousal $(\alpha=0.99)$, and orgasmic function $(\alpha=0.97)$ over the previous 24 hours. These high internal consistency values indicated that the structural integrity of the scales was not compromised by altering the recall period. Furthermore, construct validity remained uncompromised as indicated by similar correlation values found between these subscales when using daily recall (see presented correlations found in this same sample reported in Kalmbach et al. [23]) and 4-week recall (see similarly aged women's correlations in Kalmbach et al. [24]). Each scale in the present study was scored using raw scores with higher scores indicating better sexual function. Items specific to sexual activity were provided with the response choice of "No sexual activity," which was based on Meyer-Bahlburg and

\footnotetext{
${ }^{1}$ These responses were treated as missing data. For individuals who had less than $25 \%$ missing data in a given scale, proration using participants' mean scores (within that same scale) was used to estimate total factor scores. However, individuals' scale scores were treated as missing if more than $25 \%$ of data in a factor were missing. The rationale was that treating a response of "No sexual activity" as zero would artificially bias scores into indicating higher dysfunction, whereas proration allows us to estimate the total scale score based on their other responses in the same scale. However, we decided to only prorate when the response rate was $75 \%$ and above as to minimize the impact of our estimation on the data.
}

Dolezal's critique of Female Sexual Function Index (FSFI) scoring procedures [25].

The FSFI [26] is a 19-item self-report measure of female sexual response that was modified for daily use and has been validated for use in normative samples $[22,27,28]$. For the present report, we examined daily experiences of female genital response using the lubrication scale $(\alpha=0.96)$. The high internal consistency value indicated that the structural integrity of the scale was not compromised by altering the recall period. Furthermore, construct validity remained uncompromised as indicated by similar correlation values found between this subscale and those of the PFSF when using daily recall (see presented correlations found in this same sample reported in Kalmbach et al. [23]) and 4-week recall (see similarly aged women's correlations in Kalmbach et al. [24]). This scale was scored using raw scores with higher scores indicating better genital response. Based on the recommendations of Meyer-Bahlburg and Dolezal [25], FSFI items were presented with a "No sexual activity" response option. ${ }^{1}$

Three dichotomous items were used for participants to indicate presence of menstruation and occurrence of partnered sexual activity and selfstimulation. Specifically, participants were asked each day "Have you menstruated over the past 24 hours?" and responded either "Yes" (coded as 1) or "No" (coded as 2). Regarding sexual activity, participants were asked "Did you have sex (oral, anal, hand, vaginal, etc.) with another person within the past 24 hours?" and "Did you masturbate within the past 24 hours?" and responded either "Yes" (coded as 1 ) or "No" (coded as 0 ).

The present study used three items from the Pittsburgh Sleep Quality Index [29] to assess nightly levels of TST, SOL, and subjective SQ. After waking each morning, participants rated the "quality of sleep last night" and responded to items asking "How many hours of sleep did you get last night?" and "How long did it take you to fall asleep last night?" Participants reported SOL in minutes, TST in hours (to the tenths place), and SQ was measured on a 1-4 Likert-type scale with higher scores indicating better SQ.

The Positive and Negative Affect ScheduleExpanded Form (PANAS-X) [30] was used to measure fatigue and affect balance. To minimize the time demand of the daily assessments, administration of the PANAS-X was limited to seven subscales assessing daily levels of fatigue, positive affect (PA) (joviality [e.g., happy], self-assurance [e.g., proud], and serenity [e.g., calm]), and 
negative affect (NA) (fear [e.g., afraid], sadness [e.g., sad], and hostility [e.g., angry]). To create the affect balance variable, we first computed both (i) the mean item rating among PA items; and (ii) the mean item rating among NA items. Then, a ratio was created to serve as the affect balance variable $\mathrm{x}_{\mathrm{PA}}^{-} \mathrm{x}_{\mathrm{NA}}^{-}$. Of note, item means were used rather than total PA and NA scores as the ranges of PA and NA were not equal. Affect balance scores greater than 1.00 indicate a greater ratio of positive to negative affect, whereas scores less than 1.00 indicate a greater ratio of negative to positive affect. Scores close to 1.00 would indicate experiencing relatively equal amount of PA and NA.

\section{Data Analysis}

The data collected involved repeated assessments. To account for the time-nested structure of the data, analyses were conducted using hierarchical linear modeling (HLM; also known as multilevel modeling). HLM allows for the simultaneous examination of inter-individual differences and intra-individual change. As such, this analytic approach can test the predictive qualities of baseline sexual distress and age (Level 2 predictors, i.e., inter-individual differences) as well as nightly sleep parameters and daily affect balance and presence of menstruation (Level 1 predictors, i.e., intraindividual changes). In order to evaluate the temporal precedence of sleep on next-day sexual functioning, sleep predictors were lagged in the HLM models (i.e., Sleep at time $t-1$ predicting Sexual Response at time $t$ ).

\section{Preliminary Analyses}

To determine relevant covariates in predicting female sexual function (for individual $i$ at time $t$ ), each outcome variable was predicted by baseline sexual distress, depression, anxiety, oral contraceptive use, and age (for individual $i$ ), and daily affect balance and presence of menstruation (for individual $i$ at time $t$ ). These analyses were conducted to determine which variables to use as covariates in the models testing our substantive hypotheses.

Example $1^{2}$ :

$$
\begin{aligned}
\text { Desire }_{i \mathrm{it}}= & \beta_{0 \mathrm{i}}+\beta_{1} \text { Sexual distress }_{\mathrm{i}}+\beta_{2} \text { Age }_{\mathrm{i}} \\
& +\beta_{3} \text { Affect balance }_{\mathrm{it}}+\beta_{4} \text { Menstruation }_{\mathrm{it}} \\
& +\beta_{5} \text { Fatigue }_{\mathrm{it}}+\beta_{6} \text { Depression }_{\mathrm{i}} \\
& +\beta_{5} \text { Anxiety }_{\mathrm{i}} \\
& +\beta_{5} \text { Oral contraceptive use }_{\mathrm{i}}+\zeta_{0 \mathrm{i}}+\varepsilon_{\mathrm{it}}
\end{aligned}
$$

${ }^{2} \beta=$ Predictor coefficients; $\zeta=$ Level 2 stochastic part; $\varepsilon=$ Level 1 stochastic part; $i=$ individual; $t=$ time.
Example 1 examined differences in sexual function outcomes for women with varying degrees of baseline sexual distress, depression, and anxiety. Additionally, this model evaluated any daily influence of menstruation, affect balance, and fatigue on sexual response, as well as effects of age and oral contraceptives. Subsequent analyses included significant predictors as covariates to allow for the testing of relationships between sleep and sexual function independent of fatigue, affect balance, age effects, and impact of daily menstruation.

\section{Substantive Hypotheses}

To examine the influence of sleep on sexual response and behavior, sexual response indices were regressed on the previous night's sleep (i.e., lagged predictors) while controlling for relevant covariates.

Example 2:

$$
\begin{aligned}
\text { Desire }_{\mathrm{it}}= & \beta_{0 \mathrm{i}}+\beta_{1} \mathrm{TST}_{\mathrm{it}-1}+\beta_{2} \mathrm{SOL}_{\mathrm{it}-1}+\beta_{3} \mathrm{SQ}_{\mathrm{it}-1} \\
& +\beta_{4} \ldots \text { Covariates }_{\mathrm{i}(\mathrm{t})}+\zeta_{0 \mathrm{i}}+\varepsilon_{\mathrm{it}}
\end{aligned}
$$

Finally, to examine sleep's influence on sexual behavior, sexual activity and self-stimulation (dichotomous variables) were regressed on the previous night's sleep parameters and relevant covariates using multilevel mixed-effects logistic regression.

Example 3:

$$
\begin{aligned}
\text { Sexual Activity }_{\mathrm{it}}= & \beta_{0 \mathrm{i}}+\beta_{1} \mathrm{TST}_{\mathrm{it}-1}+\beta_{2} \mathrm{SOL}_{\mathrm{it}-1} \\
& +\beta_{3} \mathrm{SQ}_{\mathrm{it}-1}+\beta_{4} \ldots \text { Covariates }_{\mathrm{i}(\mathrm{t})} \\
& +\zeta_{0 \mathrm{i}}+\varepsilon_{\mathrm{it}}
\end{aligned}
$$

\section{Results}

\section{Preliminary Analyses}

According to the FSDS-R ${ }^{3}, 19.30 \%$ of women in the present study indicated clinically significant levels of sexual distress (19.81 \pm 11.24$)$. Regarding sexual activity, women reported self-stimulation on $8.90 \%$ of days and sexual activity with a partner on $24.53 \%$ of days (the latter was calculated only among women who reported having a significant other at baseline). Healthy sexual functioning was indicated for subjective sexual arousal (17.19 \pm 2.15 , range: $3-18)$, genital response $(18.57 \pm 2.55$,

${ }^{3}$ Unlike the original $0-4$ scale, the present study administered the FSDS-R using a 1-6 scale. As such, a clinical cutoff of 27 (calculated to be most parallel to the 11-point cutoff) was used to adjust for the scaling difference. 
Table 2 Zero-order correlations between female sexual response indices and predictors

\begin{tabular}{lccrr}
\hline & Desire & $\begin{array}{l}\text { Subjective } \\
\text { arousal }\end{array}$ & \multicolumn{1}{l}{$\begin{array}{l}\text { Vaginal } \\
\text { lubrication }\end{array}$} & $\begin{array}{r}\text { Orgasmic } \\
\text { function }\end{array}$ \\
\hline TST & 0.06 & 0.13 & 0.14 & 0.13 \\
SOL & -0.04 & -0.13 & -0.01 & -0.05 \\
SQ & 0.09 & 0.10 & 0.13 & 0.25 \\
Affect balance & 0.18 & 0.14 & 0.13 & 0.19 \\
Fatigue & -0.05 & -0.08 & -0.02 & -0.11 \\
Depression & -0.04 & -0.14 & -0.06 & -0.21 \\
Anxiety & -0.04 & -0.13 & -0.08 & -0.17 \\
Age & -0.05 & -0.17 & -0.19 & -0.13 \\
Sexual distress & 0.05 & -0.23 & -0.15 & -0.22 \\
\hline
\end{tabular}

Correlations are presented for descriptive purposes only; thus, no significant values are shown

Affect balance and fatigue were measured using the Positive and Negative Affect Schedule-Revised. Depression was measured using the Center for Epidemiological Studies Depression Scale. Anxiety was measured using the State-Trait Anxiety Inventory-Revised. Sexual distressed was measured using the Female Sexual Distress Scale-Revised

$\mathrm{SOL}$ = sleep onset latency; $\mathrm{SQ}=$ sleep quality; $\mathrm{TST}$ = total sleep time

range: 4-20), and orgasmic function $(20.46 \pm 3.93$, range: 4-24) as evidenced by each scale's mean being within 1 standard deviation (SD) of the maximum scale value. Average daily sexual desire was reported as within normal limits (24.71 \pm 10.45, range: $9-54$ ) as indicated by the mean being approximately in the middle of range of values. Regarding sleep parameters, participants reported good SQ $(3.09 \pm 0.38,1-4)$ with an average SOL of 21 minutes ( \pm 13.88 minutes) and an average TST of 7 hours and 22 minutes ( \pm 52 minutes). In examining prevalence of clinical levels of depression and anxiety, $3 \%$ of participants reported clinically significant levels of baseline depression (CESD: $13.37 \pm 9.78$ ) [31], whereas 9\% of participants indicated clinical levels of anxiety (STAIXS: $41.92 \pm 10.98$ ) [32]. As an initial step in examining the data, we ran zero-order correlations between sexual response indices and predictor variables for descriptive purposes (see Table 2).

Prior to conducting analyses for the substantive hypotheses, we examined whether observed intra-individual variance in our outcome variables supported our use of HLM analyses. We first ran a null model with sexual desire regressed on an intercept, a Level 1 (intra-individual) residual, and a Level 2 (inter-individual) residual (see Singer and Willett [33]). Results indicated that approximately $54 \%$ of the total variance in sexual desire was due to day-to-day fluctuations (i.e., intra-individual variations), whereas the remaining $46 \%$ was due to between-subjects factors (i.e., inter-individual differences). Thus, the data revealed marked variations in daily levels of desire to justify the examination of timevarying outcomes. Similarly, null models for subjective arousal (44\%), genital response (48\%), and orgasmic function (43\%) revealed marked day-to-day changes, thus supporting our statistical approach.

\section{Sleep Predicting Sexual Response}

To test our substantive hypotheses, we first estimated sexual desire as predicted by age, sexual distress, oral contraceptive use, baseline depression and anxiety, and daily affect balance, fatigue, and menstruation (see Table 3 ). Analyses revealed affect balance $(P<0.001)$, menstruation $(P<0.01)$, and oral contraceptive use $(P=0.03)$ to be significant predictors. Specifically, greater PA balance and oral contraceptive use were associated with higher levels of desire, whereas women desired less sex when menstruating. We then estimated desire as predicted by TST, SOL, and SQ, controlling for affect balance, menstruation, and oral contraceptive use (see Table 3). Analyses revealed that longer sleep duration predicted greater next-day sexual desire $(P=0.02)$. This effect was independent of the influence of both affect balance $(P<0.001)$, menstruation $(P=0.01)$, and contraceptive use $(P=0.04)$.

Next, we examined subjective arousal and identified baseline sexual distress $(b=-0.05, \mathrm{z}=-2.74$, $P<0.01)$ and age $(\mathrm{b}=-11, \mathrm{z}=-2.23, P=0.03)$ as relevant covariates such that greater sexual distress and older age were related to poorer subjective arousal. However, estimation of subjective arousal as predicted by sleep parameters revealed no significant findings (all $P$ values $>0.05$ ).

We then estimated daily genital response, which showed older age was associated with greater difficulty with vaginal lubrication $(P<0.01)$, but that a more PA balance was associated with better genital arousal during sexual activity $(P=0.01$; see Table 4). Analyses then revealed that longer TST the prior night was associated with poorer vaginal 
Table 3 The influence of sleep on next-day sexual desire

\begin{tabular}{|c|c|c|c|c|c|}
\hline Outcome & Predictor & $\mathrm{b}$ & z & $P$ value & $\chi^{2}(P$ value $)$ \\
\hline \multicolumn{6}{|c|}{$\begin{array}{l}\text { Determining covariates } \\
(\mathrm{N}=171, \text { Obs }=2,156)\end{array}$} \\
\hline \multirow[t]{11}{*}{ Desire $_{i t}$} & Level 1 & & & & $108.75(<0.001)$ \\
\hline & Intercept & 16.40 & 3.69 & $<0.001$ & \\
\hline & Menstruation $_{\text {it }}$ & 1.48 & 2.79 & $<0.01$ & \\
\hline & Affect balance $_{\text {it }}$ & 13.01 & 8.96 & $<0.001$ & \\
\hline & Fatigue $_{i t}$ & -0.18 & -1.68 & 0.09 & \\
\hline & Level 2 & & & & \\
\hline & Sexual Distress ${ }_{i}$ & 0.08 & 1.49 & 0.14 & \\
\hline & $\mathrm{Age}_{\mathrm{i}}$ & -0.23 & -1.41 & 0.16 & \\
\hline & Depression $_{\mathrm{i}}$ & 0.04 & 0.49 & 0.63 & \\
\hline & Anxiety $_{i}$ & -0.01 & -0.08 & 0.93 & \\
\hline & Oral contraceptives & 2.46 & 2.23 & 0.03 & \\
\hline \multicolumn{6}{|c|}{$\begin{array}{l}\text { Testing hypotheses } \\
(\mathrm{N}=171, \text { Obs }=1,859)\end{array}$} \\
\hline \multirow[t]{9}{*}{ Desire $_{i t}$} & Level 1 & & & & $89.98(<0.001)$ \\
\hline & Intercept & 11.82 & 6.13 & $<0.001$ & \\
\hline & Total sleep time it-1 & 0.32 & 2.31 & 0.02 & \\
\hline & Sleep onset latency $y_{i-1}$ & 0.01 & 1.26 & 0.21 & \\
\hline & Sleep quality $\mathrm{it}_{1-1}$ & -0.19 & -0.58 & 0.56 & \\
\hline & Menstruation $_{\text {it }}$ & 1.42 & 2.47 & 0.01 & \\
\hline & Affect balance $_{i t}$ & 12.80 & 8.58 & $<0.001$ & \\
\hline & Level 2 & & & & \\
\hline & Oral contraceptives & 2.23 & 2.03 & 0.04 & \\
\hline
\end{tabular}

In multilevel modeling, the chi-square $\left(\chi^{2}\right)$ statistic reflects the difference between the tested model and a null model with no predictors. Thus, a significant chi-square is desirable, as it is indicative of a model that accounts for significant variance in the outcome. This is in contrast to the use of chi-square in structural equation modeling, in which significant chi-square statistics are undesirable and reflect a lack of model fit. $b=$ unstandardized beta coefficient. $z$-scores represent testing the predictor's significance. Desire was measured by the Profile of Female Sexual Function. Affect balance and fatigue were measured by the Positive and Negative Affect Schedule-Expanded Form. Sexual distress was measured by the Female Sexual Distress Scale-Revised. Depression was measured using the Center for Epidemiological Studies Depression Scale. Anxiety was measured using the State-Trait Anxiety Inventory-State scale. Total sleep time, sleep onset latency, and sleep quality were measured using modified items from the Pittsburgh Sleep Quality Index

lubrication $(\mathrm{b}=-0.15, \mathrm{z}=-2.22, P=0.03)$. As this result was unexpected and contrary to prior research [12] as well as to our bivariate descriptive correlation between TST and vaginal lubrication (see Table 2), we followed up this analysis by regressing genital response on only TST to rule out multicollinearity artifact. Longer sleep duration once again predicted poorer genital response $(P=0.02)$ in the single-predictor model. Again, given the descriptive correlation showing a positive relationship, this finding was unexpected. We then examined if the relationship between TST and vaginal lubrication was different for withinperson changes (i.e., intra-individual change, Level 1 analysis) than between-person differences (inter-individual differences, Level 2 analysis). To test this possibility, mean TST was calculated for each participant, thus creating a Level 2 predictor. Descriptive analyses revealed that participants' average sleep duration over the 2-week protocol was 7 hours and 22 minutes ( \pm 50 minutes). We re-ran the model examining both the previous night's sleep duration (Level 1 predictor) and average TST for that person (Level 2 predictor), along with all relevant covariates, to estimate vaginal lubrication. Interestingly, both TST predictors were significant (see Table 4). Irrespective of average sleep duration, women reported better genital response on days following a night of shorter duration of sleep (Level 1 analysis; $P<0.01)$. In contrast, women who slept longer on average reported better genital response than women who averaged shorter durations of sleep (Level 2 analysis; $P=0.03$ ).

We then found age $(b=-0.19, \mathrm{z}=-2.27, P=$ $0.02)$, baseline sexual distress $(\mathrm{b}=-0.19, \mathrm{z}=-2.61$, $P<0.05)$, and daily affect balance $(\mathrm{b}=2.33$, $\mathrm{z}=2.80, P<0.01)$ to be relevant covariates when predicting ability to reach orgasm. Follow-up analysis, however, did not reveal an influence of sleep on next-day orgasmic function (all $P$ values $>0.05)$.

\section{Sleep Predicting Next-Day Sexual Behavior}

Multilevel logistic regression was used to examine whether sleep parameters were associated with next-day self-stimulation and partnered sexual activity. In predicting next-day self-stimulation, women with greater baseline sexual distress were more likely to masturbate (odds ratio $=1.06$, 
Table 4 Sleep predicting next-day genital response

\begin{tabular}{|c|c|c|c|c|c|}
\hline Outcome & Predictor & $\mathrm{b}$ & z & $P$ value & $\begin{array}{l}\chi^{2} \\
(P \text { value })\end{array}$ \\
\hline \multicolumn{6}{|c|}{$\begin{array}{l}\text { Determining covariates } \\
(n=137, \text { Obs }=473)\end{array}$} \\
\hline \multirow[t]{11}{*}{ Lubrication $_{\text {it }}$} & Level 1 & & & & $22.79(<0.01)$ \\
\hline & Intercept & 20.79 & 11.71 & $<0.001$ & \\
\hline & Menstruation $_{\text {it }}$ & -0.31 & -0.86 & 0.39 & \\
\hline & Affect balance $_{i t}$ & 1.96 & 2.59 & 0.01 & \\
\hline & Fatigue $_{i t}$ & 0.07 & 1.23 & 0.22 & \\
\hline & Level 2 & & & & \\
\hline & Sexual distress $s_{i}$ & -0.03 & -1.63 & 0.10 & \\
\hline & $\mathrm{Age}_{\mathrm{i}}$ & -0.15 & -2.84 & $<0.01$ & \\
\hline & Depression $_{i}$ & 0.02 & 0.71 & 0.48 & \\
\hline & Anxiety & -0.01 & -0.38 & 0.71 & \\
\hline & Oral contraceptives $i$ & 0.30 & 0.73 & 0.47 & \\
\hline \multicolumn{6}{|c|}{$\begin{array}{l}\text { Testing hypotheses } \\
(n=122, \text { Obs }=407)\end{array}$} \\
\hline \multirow[t]{9}{*}{ Lubrication $_{\text {it }}$} & Level 1 & & & & $28.61(<0.001)$ \\
\hline & Intercept & 18.37 & 8.11 & $<0.001$ & \\
\hline & Total sleep time ${ }_{i t-1}$ & -0.19 & -2.63 & $<0.01$ & \\
\hline & Sleep onset latency ${ }_{\mathrm{it}-1}$ & 0.00 & 0.22 & 0.83 & \\
\hline & Sleep quality ${ }_{\mathrm{it}-1}$ & -0.06 & -0.39 & 0.70 & \\
\hline & Affect balance $_{\text {it }}$ & 1.37 & 2.46 & 0.01 & \\
\hline & Level 2 & & & & \\
\hline & Total sleep time average $\mathrm{e}_{\mathrm{i}}$ & 0.54 & 2.18 & 0.03 & \\
\hline & $\mathrm{Age}_{\mathrm{i}}$ & -0.17 & -2.98 & $<0.01$ & \\
\hline
\end{tabular}

Lubrication was measured by the Female Sexual Function Index. Affect balance and fatigue were measured by the Positive and Negative Affect ScheduleExpanded Form. Sexual distress was measured by the Female Sexual Distress Scale-Revised. Depression was measured using the Center for Epidemiological Studies Depression Scale. Anxiety was measured using the State-Trait Anxiety Inventory-State scale. Total sleep time, sleep onset latency, and sleep quality were measured using modified items from the Pittsburgh Sleep Quality Inventory

$\mathrm{z}=3.64, P<0.001)$. No other covariates or sleep parameters were significant (all $P$ values $>0.05$ ).

Lastly, we predicted the occurrence of partnered sexual activity among women who indicated having a significant other at baseline (see Table 5). Menstruation $(P<0.01)$ and affect balance $(P<0.01)$ were identified as relevant covariates such that women were more than twice as likely to engage in partnered sexual activity when they were not menstruating and that they were nearly eight times more likely to engage in sexual activity with a partner when their affect balance ratio score increased by one point. We then examined sleep's influence on sexual activity and found that sleep duration predicted a greater likelihood of engaging in sexual activity with a partner such that a 1 hour increase in TST corresponded to a $14 \%$ greater likelihood of engaging in partnered sexual activity $(P<0.05)$.

\section{Discussion}

The present study used a daily diary approach over a 2-week period to examine prospectively how changes in nightly sleep duration, SQ, and SOL correspond to variations in female sexual response and sexual activity. Analyses revealed the impor- tance of sleep duration in healthy sexual desire and genital response, as well as partnered sexual activity. Observing that sleep duration played an important role in the regulation of sexual desire and genital arousal in a sample of young, healthy women highlights the influence of sleep on sexual functioning and behavior.

The impact of sleep duration on female sexual response was both consistent and robust to other daytime factors. In support of Andersen and colleagues' hypothesis [12], sleep duration was critical to next-day sexual desire. This relationship was independent of affect balance and fatigue, indicating a direct influence of sleep on sexual response that is not better accounted by other sleep-related daytime sequelae.

Sleep duration was also an important determinant of genital response. However, this relation was more complex than TST's influence on desire. The relationship between sleep duration and vaginal lubrication differed when examining how sleep duration predicted next-day genital arousal within women (Level 1 analysis) as compared with testing differences in genital arousal between women with varying levels of average sleep duration (Level 2 analysis). Specifically, we found that women with greater average sleep duration 
Table 5 The influence of sleep on next-day partnered sexual activity among women with significant others at baseline

\begin{tabular}{|c|c|c|c|c|c|}
\hline Outcome & Predictor & OR & z & $P$ value & $\begin{array}{l}\chi^{2} \\
(P \text { value })\end{array}$ \\
\hline \multicolumn{6}{|c|}{$\begin{array}{l}\text { Determining covariates } \\
(\mathrm{n}=86, \text { Obs }=1,092)\end{array}$} \\
\hline \multirow[t]{10}{*}{ Sexual Activity } & Level 1 & & & & \multirow{10}{*}{13.27} \\
\hline & Menstruation $_{\text {it }}$ & 2.67 & 3.42 & $<0.01$ & \\
\hline & Affect balance & 7.90 & 3.26 & $<0.01$ & \\
\hline & Fatigue $_{i t}$ & 0.93 & -1.47 & 0.14 & \\
\hline & Level 2 & & & & \\
\hline & Sexual distress & 0.98 & -0.80 & 0.43 & \\
\hline & $\mathrm{Age}_{\mathrm{i}}$ & 0.99 & -0.38 & 0.71 & \\
\hline & Depression $_{\mathrm{i}}$ & 1.02 & 0.60 & 0.55 & \\
\hline & Anxiety $_{i}$ & 1.01 & 0.52 & 0.60 & \\
\hline & Oral contraceptives & 1.59 & 1.40 & 0.16 & \\
\hline \multicolumn{6}{|c|}{$\begin{array}{l}\text { Testing hypotheses } \\
(\mathrm{n}=86, \text { Obs }=953)\end{array}$} \\
\hline \multirow[t]{6}{*}{ Sexual Activity } & Level 1 & & & & \multirow[t]{6}{*}{22.01} \\
\hline & Total sleep time $\mathrm{it}_{\mathrm{i}-1}$ & 1.14 & 1.98 & $<0.05$ & \\
\hline & Sleep onset latency $y_{\mathrm{it}-1}$ & 0.99 & -1.08 & 0.28 & \\
\hline & Sleep quality $\mathrm{it}_{\mathrm{t}-1}$ & 0.87 & -0.99 & 0.32 & \\
\hline & Menstruation $_{\text {it }}$ & 2.37 & 2.72 & 0.01 & \\
\hline & Affect balance $_{\text {it }}$ & 5.59 & 3.13 & $<0.01$ & \\
\hline
\end{tabular}

Affect balance and fatigue were measured by the Positive and Negative Affect Schedule-Expanded Form. Sexual distress was measured by the Female Sexual Distress Scale-Revised. Depression was measured using the Center for Epidemiological Studies Depression Scale. Anxiety was measured using the State-Trait Anxiety Inventory-State scale. Total sleep time, sleep onset latency, and sleep quality were measured using modified items from the Pittsburgh Sleep Quality Inventory

$\mathrm{OR}=$ odds ratio

reported better genital arousal compared with women with lower average sleep duration. This finding is consistent with prior research [12] supporting sufficient sleep duration for the promotion of healthy sexual functioning. In contrast, when women had short duration of sleep, they experienced better genital arousal the following day. Though these relationships are in opposing directions, they are not mutually exclusive. Rather, these relations may reflect the differential effects on daytime functioning of a single night vs. chronic nights of sleep deprivation.

Regarding sexual behavior, sleep duration predicted greater likelihood of next-day partnered sexual activity among our participants in romantic relationships such that an additional hour of TST corresponded to a $14 \%$ increased chance of sexual activity among our subsample of partnered individuals.

The present study demonstrated sleep duration to be important to sexual response and behavior. However, future research is necessary to identify mechanisms facilitating this association. Andersen et al. [12] posited that sleep loss led to hormonal changes, which then manifested as poorer sexual functioning. This theory suggests that deficient androgen levels mediate the impact of insufficient core sleep on sexual response. However, future research is necessary to directly test this hypothesis.
Though TST fails to capture aspects of sleep beyond duration, it may serve as a rough proxy for sleep architectural changes. For instance, shorter sleep duration is associated with markedly decreased time in REM sleep, as greater amounts of REM sleep are obtained in the latter portion of the sleep period [34]. Given that vaginal vasocongestion increases during REM sleep [6], it is possible that specific changes (within individuals) or differences (between individuals) in REM sleep may underlie the two different relationships between genital arousal and nightly and habitual sleep duration. However, as it is presently unclear whether REM sleep serves a function for female genital arousal or if REM-related vaginal vasocongestion is simply a marker of healthy genital response, the role of REM sleep in human sexual response is presently unclear. Comparing polysomnogram (PSG) profiles between women with clinical sexual dysfunction and healthy controls may provide insight into potential sleep architectural differences between these two groups. Furthermore, PSG comparison allows for testing any group differences in objectively measured sleep disorders, such as sleep-related breathing disorders.

Importantly, sleep duration may also serve as a proxy for sleep maintenance. Difficulty maintaining sleep is characterized by frequent nighttime awakenings, significant amount of time awake following initial sleep onset, and early morning 
awakenings (i.e., unintentionally waking before the desired wake time followed by an inability to re-initiate sleep). As such, it may be that fragmented, difficult-to-maintain sleep is a driving force behind sleep duration and sexual difficulties. Notably, fragmented sleep is a hallmark feature of obstructive sleep apnea [35]. Thus, the inability to maintain sleep may be an important driving force behind both the relationships between obstructive sleep apnea and sexual dysfunction, as well as sleep duration and sexual function. It may behoove researchers to explore these possibilities in future investigations.

Though the specific underlying mechanisms are presently unknown, it appears that sleep loss begets poorer sexual desire and reduced sexual activity. Further, women with chronically insufficient sleep may be at greater risk for genital arousal difficulties, though one night of sleep loss appears to lead to short-term improvement in genital arousal the following day. Given that insomnia is the most prevalent clinical sleep disorder with rates of $10 \%$ in the adult U.S. population $[36,37]$ and is characterized by persistent nocturnal symptoms of difficulty initiating and maintaining sleep [38], insomnia may pose an important risk factor for sexual dysfunction. Notably, insomnia disorder also consists of daytime impairment (e.g., fatigue, irritability, concentration difficulties) because of nocturnal symptoms. If the inability to maintain sleep contributed to sleep duration reports in the present study, then impaired sexual response following sleep loss may constitute a daytime sequela of insomnia. To our knowledge, the comorbidity between insomnia and female sexual dysfunction has not been studied. Based on our findings, the potential etiological role of insomnia in female sexual dysfunction may present a largely untapped and potentially rich area for future investigation.

Findings of the present study must be interpreted in light of some methodological limitations. A notable consideration regards the nature of our sample and that individuals were not screened for sleep disorders or use of sleep aids. College students are known for having irregular sleep schedules and greater prevalence of delayed sleep/wake patterns [39]. Further, participants were not screened for psychiatric conditions other than depression and anxiety, chronic medical conditions, medication use (other than antidepressants and oral contraceptives), or sleeping environmental factors, thus we cannot account for their potential influences. Additionally, our sample reported healthy sexual functioning over the 2-week period (all sexual response scale means were within $1 \mathrm{SD}$ of the maximum value of the scale, except desire). As such, range restriction may have impeded our ability to detect statistically significant relations between sleep parameters and sexual functioning and limits generalizability to individuals with sexual dysfunction. Moreover, though participants reported healthy sexual response over the 2 -week study, it is difficult to compare their sexual health with samples in other studies, as it is unclear whether aggregated daily sexual response ratings can be compared validly with ratings of longer recall periods such as month-long recall. Thus, though the ratings appeared to reflect healthy sexual response in this sample, we caution against drawing decisive clinical impressions based on daily sexual functioning ratings. Further, regarding measurement of study variables, though selfreport is an important and useful method for collecting sleep data, the gold standard of assessing sleep parameters involves the use of both subjective and objective (i.e., PSG, actigraphy) measures.

In addition to our findings regarding sleep and sexual response, we found a number of relationships between daily affect balance and same-day sexual functioning. PA balance (i.e., greater ratio of $\mathrm{PA}$ to $\mathrm{NA}$ ) was a robust predictor of sexual desire and ability to achieve orgasm, as well as self-stimulation and partnered sexual activity. In comparison, NA balance (i.e., greater ratio of NA to PA) was related to poorer genital response. Affect balance was not associated with subjective arousal. Though prior studies have shown that specific affect states are important to daily sexual response [40], this investigation suggests that the balance of these affect states is meaningfully related to daily desire, genital response, orgasmic function, and sexual behavior.

\section{Conclusions}

Good sleep is important for maintaining healthy sexual functioning. Decreases in desire and genital response, as well as reduced likelihood of partnered sexual activity, are predicted by both nightly and habitual sleep duration. Notably, these effects were independent of age, sexual distress, daytime affect and fatigue, or presence of menstruation. These findings suggest that acute sleep disturbance may contribute to sexual complaints and reduced sexual activity. Future research may benefit from taking a more comprehensive approach to examining sleep parameters by using both subjective and objective measures. 
Additionally, the relationship between insomnia and sexual dysfunction may prove to be an overlooked and important area of interest for clinical research. Clinicians may consider assessing patients' sleep habits and insomnia symptoms as potential factors influencing sexual difficulties.

Corresponding Author: David A. Kalmbach, PhD, Sleep and Circadian Research Laboratory, Department of Psychiatry, University of Michigan Medical School, Rachel Upjohn Building, 4250 Plymouth Rd, Ann Arbor, MI 48105, USA. Tel: 330-204-6942; Fax: 734936-7868; E-mail: dkalmbac@gmail.com

Conflict of Interest: The authors report no conflicts of interest.

\section{Statement of Authorship}

\section{Category 1}

(a) Conception and Design

David A. Kalmbach; Jeffrey A. Ciesla

(b) Acquisition of Data

David A. Kalmbach

(c) Analysis and Interpretation of Data

David A. Kalmbach

\section{Category 2}

(a) Drafting the Manuscript

David A. Kalmbach

(b) Revising It for Intellectual Content

David A. Kalmbach; Vivek Pillai; J. Todd Arnedt; Jeffrey A. Ciesla

\section{Category 3}

(a) Final Approval of the Completed Manuscript David A. Kalmbach; J. Todd Arnedt; Vivek Pillai; Jeffrey A. Ciesla

\section{References}

1 Lewis RW, Fugl-Meyer KS, Bosch R, Fugl-Meyer A, Laumann EO, Martin-Morales A. Epidemiology/risk factors of sexual dysfunction. J Sex Med 2004;1:35-9.

2 Laurent SM, Simons AD. Sexual dysfunction in depression and anxiety: Conceptualizing sexual dysfunction as part of an internalizing dimension. Clin Psychol Rev 2009;29:573-85.

3 Fabre LF, Smith LC. The effect of major depression on sexual function in women. J Sex Med 2012;9:231-9.

4 Rosen RC. Prevalence and risk factors of sexual dysfunction in men and women. Curr Psychiatry Rep 2000;2:189-95.

5 Abel GG, Murphy WD, Becker JV, Bitar A. Women's vaginal responses during REM sleep. J Sex Marital Ther 1979; 5:5-14.

6 Fisher C, Cohen HD, Schiavi RC, David D, Furman B, Ward $\mathrm{K}$, Edwards A, Cunningham J. Patterns of female sexual arousal during sleep and waking: Vaginal thermo-conductance studies. Arch Sex Behav 1983;12:97-122.

7 Vogel GW, A motivational function of REM sleep. The functions of sleep. p. 233-50. 1979.
8 Köseoğlu N, Koseoglu H, Oya I, Oztura I, Baklan B, Ikiz AO, Esen AA. Original research-Women's sexual health: Sexual function status in women with obstructive sleep apnea syndrome. J Sex Med 2007;4:1352-7.

9 Onem K, Erol B, Kadioglu P, Yalin AS, Canik U, Cuhadaroglu C, Kadioglu A. Is sexual dysfunction in women with obstructive sleep apnea-hypopnea syndrome associated with the severity of the disease? A pilot study. J Sex Med 2008;5:2600-9.

10 Petersen M, Kristensen E, Berg S, Giraldi A, Midgren B. Sexual function in female patients with obstructive sleep apnea. J Sex Med 2011;8:2560-8.

11 Taylor DJ, Mallory LJ, Durrence HH, Riedel BW, Bush AJ. Comorbidity of chronic insomnia with medical problems. SLEEP 2007;30:213-8.

12 Andersen ML, Alvarenga TF, Mazaro-Costa R, Hachul HC, Tufik S. The association of testosterone, sleep, and sexual function in men and women. Brain Res 2011;1416:80-104.

13 Dement W, Cohen H, Ferguson J, Zarcone V. A sleep researcher's odyssey: The function and clinical significance of REM sleep. In: Madow L, Snow LH, eds. The psychodynamic implications of the physiological studies on dreams. Springfield, IL: Thomas; 1970:71-123.

14 Bancroft J, Sanders D, Davidson D, Warner P. Mood, sexuality, hormones, and the menstrual cycle. III. Sexuality and the role of androgens. Psychosom Med 1983;45:509-16.

15 Regan PC. Rhythms of desire: The association between menstrual cycle phases and female sexual desire. Can J Hum Sex 1996;5:145-56.

16 Derogatis LR. The Derogatis Interview for Sexual Functioning (DISF/DISF-SR): An introductory report. J Sex Marital Ther 1997;23:291-304.

17 DeRogatis L, Clayton A, Lewis-D'Agostino D, Wunderlich G, Fu Y. Validation of the female sexual distress scale-revised for assessing distress in women with hypoactive sexual desire disorder. J Sex Med 2008;5:357-64.

18 Radloff LS. The CES-D scale a self-report depression scale for research in the general population. Appl Psych Meas 1977;1:385-401.

19 Spielberger CD, Gorssuch RL, Lushene PR, Vagg PR, Jacobs GA. Manual for the state-trait anxiety inventory. Palo Alto, CA: Consulting Psychologists Press, Inc.; 1983.

20 McHorney C, Rust J, Golombok S, Davis S, Bouchard C, Brown C, Basson R, Sarti CD, Kuznicki J, Rodenberg C, DeRogatis L. Profile of female sexual function: A patientbased, international, psychometric instrument for the assessment of hypoactive sexual desire in oophorectomized women. Menopause 2004;11:474-83.

21 DeRogatis L, Rust J, Golombok S, Bouchard C, Nachtigall L, Rodenberg C, Kuznicki J, McHorney CA. Validation of the profile of female sexual function (PFSF) in surgically and naturally menopausal women. J Sex Marital Ther 2004;30: $25-36$.

22 Kalmbach DA, Ciesla JA, Janata JW, Kingsberg SA. The validation of the female sexual function index, male sexual function index, and profile of female sexual function for use in healthy, young adults. Arch Sex Behav 2014;doi: 10.1007/ s10508-014-0334-y.

23 Kalmbach DA, Kingsberg SA, Ciesla JA. How changes in depression and anxiety symptoms correspond to variations in female sexual response in a nonclinical sample of young women: A daily diary study. J Sex Med 2014;11:2915-27.

24 Kalmbach DA, Ciesla JA, Janata JW, Kingsberg SA. Specificity of anhedonic depression and anxious arousal with sexual problems among sexually healthy young adults. Journal of Sexual Medicine 2012;9:505-13.

25 Meyer-Bahlburg HF, Dolezal C. The female sexual function index: A methodological critique and suggestions for improvement. J Sex Marital Ther 2007;33:217-24. 
26 Rosen RC, Brown C, Heiman J, Leiblum S, Meston C, Shabsigh R, Ferguson D, D'Agostino R. The female sexual function index (FSFI): A multidimensional self-report instrument for the assessment of female sexual function. J Sex Marital Ther 2000;26:191-208.

27 Carvalho J, Vieira AL, Nobre PJ. Latent structures of female sexual functioning. Arch Sex Behav 2012;41:907-17.

28 Opperman EA, Benson LE, Milhausen RR. Confirmatory factor analysis of the female sexual function index. J Sex Res 2013;50:29-36.

29 Buysse DJ, Reynolds CF, Monk TH, Berman SR, Kupfer DJ. The Pittsburgh Sleep Quality Index (PSQI): A new instrument for psychiatric research and practice. Psychiatry Res 1989; 28:193-213.

30 Watson D, Clark LA, The PANAS-X: Manual for the positive and negative affect schedule-expanded form. 1999.

31 Santor DA, Zuroff DC, Ramsay JO, Cervantes P, Palacios J. Examining scale discriminability in the BDI and CES-D as a function of depressive severity. Psychol Assess 1995;7: $131-9$.

32 Kvaal K, Ulstein I, Nordhus IH, Engedal K. The Spielberger state-trait anxiety inventory (STAI): The state scale in detecting mental disorders in geriatric patients. Int J Geriatr Psychiatry $2005 ; 20: 629-34$.
33 Singer JD, Willett JB. Applied longitudinal data analysis: modelling change and event occurrence. New York: Oxford University; 2003

34 Carskadon MA, Dement WC. Normal human sleep: An overview. In: Kryger MH, Roth T, Dement WC, eds. Principles and practice of sleep medicine. 5th edition. St. Louis, MO: Elsevier; 2011:16-26.

35 Bonnet MH. Acute sleep deprivation. In: Kryger MH, Roth T, Dement WC, eds. Principles and practice of sleep medicine. 5th edition. St. Louis, MO: Elsevier; 2011:54-66.

36 Roth T, Roehrs T. Insomnia: epidemiology, characteristics, and consequences. Clin Cornerstone 2003;5:5-15.

37 Morin CM, LeBlanc M, Daley M, Gregoire JP, Merette C. Epidemiology of insomnia: Prevalence, self-help treatments, consultations, and determinants of help-seeking behaviors. Sleep Med 2006;7:123-30.

38 American Academy of Sleep Medicine. International classification of sleep disorders. Third edition (ICSD-3). Darien, Illinois. 2014.

39 Lund HG, Reider BD, Whiting AB, Prichard JR. Sleep patterns and predictors of disturbed sleep in a large population of college students. J Adolescent Health 2010;46:124-32.

40 Kalmbach DA, Pillai V. Daily affect and female sexual function. J Sex Med 2014;11:2938-54. 